\title{
Serotype distribution and antimicrobial resistance of Streptococcus pneumoniae prior to introduction of the 10-valent pneumococcal conjugate vaccine in Brazil, 2000-2007
}

\author{
Ana Paula de O. Menezes ${ }^{\mathrm{a}, 1}$, Leila C. Campos ${ }^{\mathrm{a}, 1}$, Milena S. dos Santos ${ }^{\mathrm{a}}$, Jailton Azevedo ${ }^{\mathrm{a}}$, \\ Renan C.N. dos Santos a , Maria da Gloria S. Carvalho ${ }^{b}$, Bernard W. Beall ${ }^{b}$, Stacey W. Martin ${ }^{c}$, \\ Katia Salgado $^{d}$, Mitermayer G. Reis ${ }^{a}$, Albert I. Ko ${ }^{a, e}$, Joice N. Reis ${ }^{a, f, *}$ \\ a Centro de Pesquisas Gonçalo Moniz, Fundação Oswaldo Cruz, Salvador, BA 40296-710, Brazil \\ ${ }^{\mathrm{b}}$ Respiratory Diseases Branch, Centers for Disease Control E Prevention, Atlanta, GA 30333, USA \\ ${ }^{\mathrm{c}}$ Meningitis and Vaccine Preventable Diseases Branch, Centers for Disease Control \& Prevention, Atlanta, GA 30333, USA \\ ${ }^{\mathrm{d}}$ Hospital Couto Maia, Secretary of Health for the State of Bahia, Salvador, BA 40415-100, Brazil \\ e Department of Epidemiology and Public Health, Yale School of Medicine, New Haven, USA \\ ${ }^{\mathrm{f}}$ Faculdade de Farmácia, Universidade Federal da Bahia, Salvador, BA 40170-115, Brazil
}

\section{A R T I C L E I N F O}

\section{Article history:}

Received 23 September 2010

Received in revised form

29 November 2010

Accepted 6 December 2010

Available online 21 December 2010

\section{Keywords:}

Streptococcus pneumoniae

Vaccine

Antimicrobial resistance

Meningitis

Children

\begin{abstract}
A B S T R A C T
This study describes the serotype distribution and antibiotic resistance patterns among 397 S. pneumoniae meningitis case isolates recovered in Salvador, Brazil, during the period of 2000-2007, before introduction of the 10 -valent pneumococcal conjugate vaccine.

The active hospital-based surveillance showed a decline in the annual incidence rates of pneumococcal meningitis during the period of study, from 1.12 cases to 0.83 cases/100,000 persons for all age groups $(P<0.001)$, with an overall case-fatality rate of $28.6 \%$ ( 113 of 395$)$ for all patients and $41.9 \%$ ( 57 of 136$)$ for those $<5$ years of age. Serotypes $14(n=55 ; 13.9 \%), 3(n=32 ; 8.1 \%), 23 \mathrm{~F}(n=32 ; 8.1 \%), 19 \mathrm{~F}(n=31 ; 7.8 \%)$, $6 \mathrm{~B}(n=30 ; 7.6 \%), 18 \mathrm{C}(n=28 ; 7.1 \%)$, and $6 \mathrm{~A}(n=20 ; 5 \%)$ were the most prevalent serotypes. In patients $<5$ years the estimated projected coverage of $7-, 10$ - and 13 -valent conjugate vaccines was $74.3 \%, 75.7 \%$ and $83.1 \%$, respectively. Antimicrobial susceptibility testing revealed that $22.1 \%(n=88)$ of isolates were non-susceptible to penicillin, $56 \%$ were non-susceptible to trimethoprim/sulphamethoxazole, and $29.6 \%$ were non-susceptible to tetracycline. Nonsusceptibility to penicillin and cefotaxime was detected solely among serotype 14 isolates $(n=4 ; 1 \%)$. This study provides an important baseline to assess the impact of conjugate vaccine implantation on the epidemiology of meningitis due to Streptococcus pneumoniae in Salvador, Brazil.
\end{abstract}

(c) 2010 Elsevier Ltd. All rights reserved.

\section{Introduction}

Streptococcus pneumoniae (pneumococcus) is a major cause of meningitis, pneumonia, and bacteremia, especially among young children and older adults [1]. It is estimated that 1.6-2.2 million children die of pneumococcal disease every year in the world, primarily within developing countries. This death toll includes $0.7-1$ million children $<5$ years of age [2-5].

\footnotetext{
* Corresponding author at: Centro de Pesquisas Gonçalo Moniz, Fundação Oswaldo Cruz/MS, Rua Waldemar Falcão 121, Salvador, Bahia 40296-710, Candeal, Brazil. Tel.: +55 713176 2302; fax: +55 7131762281 .

E-mail addresses: joice@ufba.br, joice@conveniado.bahia.fiocruz.br (J.N. Reis).

1 These authors contributed equally to this work.
}

Introduction of heptavalent pneumococcal conjugate vaccine (PCV7) for infants led to substantial reductions in the incidence of invasive pneumococcal disease (IPD) in the United States and other industrialized countries [2,6]. IPD rates in the vaccine era have also decreased among unvaccinated older children, adults, and elderly persons through reductions in nasopharyngeal colonization and transmission of vaccine-type pneumococci from vaccinated children (herd effect) [7]. However, the increase in the rate of invasive pneumococcal disease (IPD) cases caused by non-vaccine strains has been a concern [6,8]. Although PCV7 continues to effectively decrease the pneumococcal disease burden in the United States, the incidence of IPD caused by serotype 19A strains has increased among vaccinated children, and these strains are often highly resistant to commonly used antimicrobials [9-11].

In Brazil, the national vaccine program using the recently licensed 10-valent PCV (PHiD-CV) vaccine [12] has been imple- 
mented in 2010. This vaccine includes three additional serotypes ( 1,5 and $7 F$ ) and is projected to cover $\geq 80 \%$ of serotypes causing invasive pneumococcal diseases (IPD) in most regions of the world $[13,14]$.

Assessment of changes in serotype distribution potentially related to vaccine introduction requires valid baseline values of both the incidence of IPD and of serotype distribution. Since December 1995, an active hospital-based surveillance for pneumococcal meningitis (PnM) has been conducted in the state reference hospital for infectious diseases in Salvador, Brazil. In this study we describe the pneumococcal serotype distribution and antibiotic resistance patterns among meningitis case isolates from patients referred to that hospital between January 2000 and December 2007. The main objective of our study was to provide a baseline for the interpretation of any changes arising from the future PCV10 vaccination strategy.

\section{Material and methods}

\subsection{Surveillance}

Since December 1995, active hospital-based surveillance for pneumococcal meningitis (PnM) has been conducted in Hospital Couto Maia, the state reference hospital for infectious diseases in Salvador, Brazil, which is responsible for about $95 \%$ of the meningitis reports from the metropolitan region [15]. Data obtained from the period of 1995-1999 and details of the surveillance study have been described previously [16,17]. For the present analysis, the study population included all patients hospitalized between 1 January 2000 and 31 December 2007 with pneumococcal meningitis, consisted of $34.4 \%$ women and $65.6 \%$ men aged $0-83$ years (mean age $18 \pm 19$ years). Cases were defined by the isolation of S. pneumoniae from cerebrospinal fluid (CSF) specimens and/or by positive latex agglutination test result from a patient with clinical signs and symptoms of meningitis. Patients were identified at the state infectious disease hospital where according to the state health secretary procedures, all suspected cases of meningitis for the region are referred for diagnostic procedures, including routine lumbar puncture and cerebrospinal fluid examination, and to implement isolation precaution protocols. A study team of physicians and medical students reviewed laboratory records five days a week to identify new culture isolations. Demographic and clinical presentations of the patients were collected during interviews or medical chart review.

\subsection{Laboratory procedures}

S. pneumoniae isolates from CSF cultures were identified by standard bacteriological techniques [18]. From 2000 to 2005, pneumococcal strains were serotyped by the Quellung reaction with type-specific antipneumococcal sera prepared at the Centers for Disease Control and Prevention (CDC). After 2006, the isolates were serotyped by multiplex-PCR as described elsewhere $[19,20]$. DNA extraction and PCR conditions were performed as described [21]. Isolates with negative or equivocal multiplex PCR results were subjected to Quellung reaction testing for capsular type definition. All isolates identified as serogroup 6 were subjected to $w_{c i N}{ }^{6 C}$-specific PCR as previously described for the identification of potential serotype 6C and 6D isolates [22]

\subsection{Antimicrobial susceptibility testing}

The broth microdilution method was performed according to Clinical and Laboratory Standards Institute recommendations [23] to determine susceptibility of the isolates to penicillin (Pen), ofloxacin (Ofl), cefotaxime (Cef), clindamycin(Clin), chloramphenicol (Clo), erythromycin (Eri), tetracycline (Tet), rifampicin (Rif), trimethoprim/sulphamethoxazole (Sxt), and vancomycin (Van) (Sigma-Aldrich, Germany). Quality control was performed by testing S. pneumoniae ATCC 49619. Isolates with a penicillin MIC value $\geq 0.12 \mu \mathrm{g} / \mathrm{mL}$ were defined as penicillin-non-susceptible. Intermediate and resistant isolates were considered non-susceptible.

\subsection{Data management}

Patients residing in Metropolitan Salvador (population, 3,120,303 inhabitants), which includes Salvador and 12 municipalities, were included for incidence calculations. Incidence calculations were based on the 2000 Brazilian census, provided by the Instituto Brasileiro de Geografia e Estatística without adjusting for population growth [24]. The population in Metropolitan region of Salvador is stable regarding birth cohort and migration rate [24]. Data were entered and managed by Epi Info version 3.5.1 (CDC, Atlanta, GA, USA). The chi-square for trend was applied to compare incidence among the seven years (2000-2007). The chi-square test or Fisher's exact test was performed to compare proportions. Differences were defined to be significant if the two-tailed $P$-value was $<0.05$.

A generalized linear model was fitted to the $\log 10$ transformed penicillin MIC values to evaluate trends over time using SAS $^{\circledR} 9.1$ for Windows (Cary, NC). Transformations were made to improve normality and to reduce the influence of outliers. The antilogarithm of model predicted values provided a nonlinear trend line through the yearly GMCs.

\subsection{Ethics}

This project was approved by the Research Ethical Committee of Fundação Oswaldo Cruz and of Hospital Couto Maia.

\section{Results}

We identified 421 cases of pneumococcal meningitis (PnM) at Hospital Couto Maia, from January 2000 to December 2007. Considering that 236 patients resided within the Metropolitan Salvador, the annual incidence rates of pneumococcal meningitis declined by $26 \%$ during the 8 -year of surveillance, ranging from 1.12 to 0.83 cases per 100,000 population (mean incidence, 0.9 cases $/ 100,000$ persons year for all age groups $[P<0.001]$ ) (Table 1 ). Mean incidence for children $<5$ years of age was 4.2 cases/100,000 persons-year (range from 6.4 to 1.78$)[P<0.001]$. There was an overall casefatality ratio of $28.6 \%$ (113 of 395) for all patients and $41.9 \%$ (57 of 136 ) for those $<5$ years of age. The majority of the $S$. pneumoniae meningitis cases were patients aged $>5$ years $(254 / 402 ; 63.2 \%)$, particularly among those aged $5-49$ years $(227 / 402 ; 56.5 \%)$ (data not shown). The other pneumococcal meningitis cases comprised children aged $<2$ years $(112 / 402 ; 27.9 \%)$, and $2-5$ years $(36 / 402 ; 9 \%)$. A higher proportion of cases were males than females (65.6\% versus $34.4 \%$, respectively).

Of 421 patients with pneumococcal meningitis, 402 had positive CSF cultures for $S$. pneumoniae and 19 had negative cultures and positive latex agglutination results for $S$. pneumoniae. From 402 isolates, 397 were available for serotyping and antimicrobial susceptibility testing. These isolates represented 43 different serotypes. The predominant serotypes were $14(n=55 ; 13.9 \%), 3$ $(n=32 ; 8.1 \%), 23 \mathrm{~F}(n=32 ; 8.1 \%), 19 \mathrm{~F}(n=31 ; 7.8 \%), 6 \mathrm{~B}(n=30 ; 7.6 \%)$, $18 \mathrm{C}(n=28 ; 7.1 \%)$, and $6 \mathrm{~A}(n=20 ; 5 \%)$ (Table 2$)$. We did not found any fluctuation in respect of yearly distribution of serotypes during the study period. The overall percentage of capsular types included in the 7-, 10-, and 13-valent vaccines accounted, respectively, for $50.9 \%, 51.9 \%$ and $67.3 \%$. 
Table 1

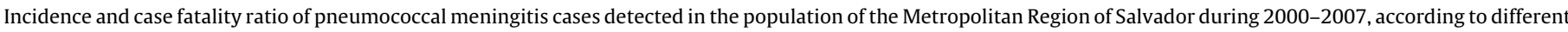
age groups.

\begin{tabular}{|c|c|c|c|c|c|c|c|c|c|c|}
\hline \multirow[t]{2}{*}{ Year } & \multicolumn{2}{|l|}{$<1$ year } & \multicolumn{2}{|l|}{$<2$ years } & \multicolumn{2}{|l|}{$2-5$ years } & \multicolumn{2}{|l|}{$>5$ years } & \multicolumn{2}{|l|}{ Total } \\
\hline & Incidence $^{a}$ & $\mathrm{CFR}^{\mathrm{b}}(\%)$ & Incidence & CFR (\%) & Incidence & CFR (\%) & Incidence & CFR (\%) & Incidence & CFR (\%) \\
\hline 2000 & 27.9 & 35.7 & 13.7 & 35.7 & 3.3 & 0 & 0.6 & 6.3 & 1.12 & 17.1 \\
\hline 2001 & 27.9 & 35.7 & 16.6 & 41.2 & 0.7 & 0 & 0.8 & 17.4 & 1.31 & 26.8 \\
\hline 2002 & 17.9 & 55.5 & 8.8 & 55.5 & 1.3 & 0 & 0.5 & 28.6 & 0.80 & 36.0 \\
\hline 2003 & 19.9 & 30.0 & 12.7 & 30.7 & 1.3 & 0 & 0.8 & 13.0 & 1.21 & 18.4 \\
\hline 2004 & 8.0 & 50.0 & 6.8 & 28.6 & 2.0 & 100.0 & 0.5 & 14.3 & 0.76 & 37.5 \\
\hline 2005 & 13.9 & 57.1 & 6.8 & 57.1 & 0.7 & 100.0 & 0.3 & 30.0 & 0.57 & 44.4 \\
\hline 2006 & 18.0 & 25.0 & 6.8 & 14.3 & 2.0 & 66.6 & 0.7 & 31.6 & 0.92 & 31.0 \\
\hline 2007 & 8.0 & 25.0 & 3.9 & 25.0 & 2.0 & 33.3 & 0.7 & 21.0 & 0.83 & 23.1 \\
\hline
\end{tabular}

a Incidence per 100,000 population.

b Case Fatality Ratio.

For 382 patients of known age, the serotype coverage of the 7-, 10- and 13-valent pneumococcal conjugate vaccines (PCVs) is shown in Fig. 1. PCV-7 projected coverage was 69.9\%, 74.7\%, 74.3\%, and $36.8 \%$ of $S$. pneumoniae isolates in patients $<1$ year, $<2$ years, $2-5$ years, $>5$ years old, respectively. PCV-10 projected coverage was $72 \%, 77.4 \%, 74.3 \%$, and $38 \%$ of isolates from patients in these age groups, respectively. The projected PCV-13 coverage was $79.6 \%$, $82.8 \%, 82.9 \%$, and $58.7 \%$ of $S$. pneumoniae isolates in patients $<1$ year, $<2$ years, $2-5$ years, $>5$ years old, respectively. In children $<5$ years of age, PCV-7, PCV-10 and PCV-13 were projected to cover $74.3 \%, 75.7 \%$, and $83.1 \%$ of the isolates, respectively. Non-vaccine serotypes were more common in patients $>5(41.3 \%)$ years of age, followed by patients $2-5$ (34.2\%) years and patients $<1(20.4 \%)$ year of age.
The antibiotic susceptibilities of the $397 \mathrm{~S}$. pneumoniae isolates are summarized in Table 2. All isolates were susceptible to vancomycin and clindamycin, and $\geq 98 \%$ of isolates were susceptible to ofloxacin, cefotaxime, erythromycin, chloramphenicol (Clo), and rifampicin. Overall, 22.1\% $(n=88)$ of isolates were non-susceptible to penicillin, 56\% $(n=223)$ to trimethoprim-sulphamethoxazole (Sxt), and 29.6\% $(n=117)$ to tetracycline. A total of $86.4 \%$ of PCV7 isolates were non-susceptible to penicillin, particularly among serotypes $14(n=37,42 \%), 23 \mathrm{~F}$ $(n=19,21.6 \%), 6 \mathrm{~B}(n=10,11.4 \%)$, and $19 \mathrm{~F}(n=9,10.2 \%)$. Few penicillin-nonsusceptible pneumococci (PNSP) were found among other serotypes included in PCV10, PCV13 and among nonvaccine serotypes. Similarly, high percentages of nonsusceptibility to trimethoprim-sulphamethoxazole and tetracycline (tet) were

Table 2

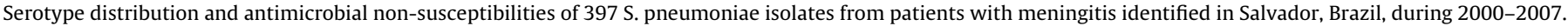

\begin{tabular}{|c|c|c|c|c|c|c|c|c|c|}
\hline Serotype & No. (\%) of isolates & Penicillin & $\begin{array}{l}\text { Trimethoprim- } \\
\text { sulphamethoxazole }\end{array}$ & Tetracycline & Ofloxacin & Cefotaxime & Erythromycin & Chloramphenicol & Rifampicin \\
\hline All Serotypes & $397(100)$ & $88(22.2)$ & $223(56)$ & $117(29.6)$ & $6(1.5)$ & $4(1.0)$ & $3(0.8)$ & $3(0.8)$ & $1(0.3)$ \\
\hline \multicolumn{10}{|c|}{ PCV7 serotypes } \\
\hline All & $203(51.1)$ & $76(86.4)$ & $134(60.1)$ & $46(39.3)$ & $3(50)$ & $4(100)$ & $1(33.3)$ & $1(33.3)$ & $0(0)$ \\
\hline 14 & $55(13.9)$ & $37(42)$ & $47(21.1)$ & $13(11.1)$ & $2(33.3)$ & $4(100)$ & $1(33.3)$ & $0(0)$ & $0(0)$ \\
\hline $6 \mathrm{~B}$ & $30(7.6)$ & $10(11.4)$ & $26(11.7)$ & $5(4.3)$ & $0(0)$ & $0(0)$ & $0(0)$ & $1(33.3)$ & $0(0)$ \\
\hline $19 \mathrm{~F}$ & $31(7.8)$ & $9(10.2)$ & $24(10.8)$ & $3(2,6)$ & $1(16.7)$ & $0(0)$ & $0(0)$ & $0(0)$ & $0(0)$ \\
\hline $18 \mathrm{C}$ & $28(7.1)$ & $0(0)$ & $9(4)$ & $3(2.6)$ & $0(0)$ & $0(0)$ & $0(0)$ & $0(0)$ & $0(0)$ \\
\hline $23 \mathrm{~F}$ & $32(8.1)$ & $19(21.6)$ & $20(9.0)$ & $18(15.4)$ & $0(0)$ & $0(0)$ & $0(0)$ & $0(0)$ & $0(0)$ \\
\hline 4 & $19(4.8)$ & $1(1.1)$ & $6(2.7)$ & $3(2.6)$ & $0(0)$ & $0(0)$ & $0(0)$ & $0(0)$ & $0(0)$ \\
\hline $9 \mathrm{~V}$ & $8(2.0)$ & $0(0)$ & $3(1.3)$ & $1(0.9)$ & $0(0)$ & $0(0)$ & $0(0)$ & $0(0)$ & $0(0)$ \\
\hline \multicolumn{10}{|c|}{ PCV10 serotypes } \\
\hline All & $208(52.4)$ & $76(86.4)$ & $136(61)$ & $47(40.2)$ & $3(50)$ & $4(100)$ & $1(33.3)$ & $1(33.3)$ & $0(0)$ \\
\hline+1 & $1(0.3)$ & $0(0)$ & $0(0)$ & $0(0)$ & $0(0)$ & $0(0)$ & $0(0)$ & $0(0)$ & $0(0)$ \\
\hline+5 & $3(0.8)$ & $0(0)$ & $2(0.9)$ & $1(0.9)$ & $0(0)$ & $0(0)$ & $0(0)$ & $0(0)$ & $0(0)$ \\
\hline$+7 \mathrm{~F}$ & $1(0.3)$ & $0(0)$ & $0(0)$ & $0(0)$ & $0(0)$ & $0(0)$ & $0(0)$ & $0(0)$ & $0(0)$ \\
\hline \multicolumn{10}{|c|}{ PCV13 serotypes } \\
\hline All & $270(68)$ & $83(94.3)$ & $162(72.6)$ & $61(51.7)$ & $4(66.7)$ & $4(100)$ & $1(33.3)$ & $2(66.7)$ & $1(100)$ \\
\hline+3 & $32(8.1)$ & $0(0)$ & $4(1.8)$ & $6(5.1)$ & $0(0)$ & $0(0)$ & $0(0)$ & $1(33.3)$ & $0(0)$ \\
\hline$+6 \mathrm{~A}$ & $20(5.0)$ & $3(3.4)$ & $15(6.7)$ & $5(4.3)$ & $1(16.7)$ & $0(0)$ & $0(0)$ & $0(0)$ & $1(100)$ \\
\hline+19 A & $10(2.5)$ & $4(4.5)$ & $7(3.1)$ & $3(2.6)$ & $0(0)$ & $0(0)$ & $0(0)$ & $0(0)$ & $0(0)$ \\
\hline \multicolumn{10}{|c|}{ Non-vaccine serotypes } \\
\hline $10 \mathrm{~A}$ & $13(3.3)$ & $0(0)$ & $5(2.2)$ & $12(10.3)$ & $0(0)$ & $0(0)$ & $0(0)$ & $0(0)$ & $0(0)$ \\
\hline $9 \mathrm{~N}$ & $10(2.5)$ & $0(0)$ & $5(2.2)$ & $8(6.8)$ & $2(33.3)$ & $0(0)$ & $1(33.3)$ & $1(33.3)$ & $0(0)$ \\
\hline $6 C$ & $10(2.5)$ & $1(1.1)$ & $8(3.6)$ & $1(0.9)$ & $0(0)$ & $0(0)$ & $1(33.3)$ & $0(0)$ & $0(0)$ \\
\hline $23 \mathrm{~B}$ & $9(2.3)$ & $2(2.3)$ & $7(3.1)$ & $2(1.7)$ & $0(0)$ & $0(0)$ & $0(0)$ & $0(0)$ & $0(0)$ \\
\hline $18 \mathrm{~A}$ & $9(2.3)$ & $0(0)$ & $1(0.4)$ & $5(4.3)$ & $0(0)$ & $0(0)$ & $0(0)$ & $0(0)$ & $0(0)$ \\
\hline $18 \mathrm{~F}$ & $8(2.0)$ & $0(0)$ & $3(1.3)$ & $3(2.6)$ & $0(0)$ & $0(0)$ & $0(0)$ & $0(0)$ & $0(0)$ \\
\hline 8 & $6(1.5)$ & $0(0)$ & $2(0.9)$ & $4(3.4)$ & $0(0)$ & $0(0)$ & $0(0)$ & $0(0)$ & $0(0)$ \\
\hline $11 \mathrm{~A}$ & $6(1.5)$ & $0(0)$ & $5(2.2)$ & $1(0.9)$ & $0(0)$ & $0(0)$ & $0(0)$ & $0(0)$ & $0(0)$ \\
\hline 13 & $5(1.3)$ & $1(1.1)$ & $2(0.9)$ & $1(0.9)$ & $0(0)$ & $0(0)$ & $0(0)$ & $0(0)$ & $0(0)$ \\
\hline $16 \mathrm{~F}$ & $6(1.5)$ & $0(0)$ & $4(1.8)$ & $1(0.9)$ & $0(0)$ & $0(0)$ & $0(0)$ & $0(0)$ & $0(0)$ \\
\hline $15 C$ & $5(1.3)$ & $0(0)$ & $4(1.8)$ & $0(0)$ & $0(0)$ & $0(0)$ & $0(0)$ & $0(0)$ & $0(0)$ \\
\hline NT & $1(0.25)$ & $0(0)$ & $1(0.4)$ & $0(0)$ & $0(0)$ & $0(0)$ & $0(0)$ & $0(0)$ & $0(0)$ \\
\hline Others $^{\mathrm{a}}$ & $39(9.8)$ & $1(1.1)$ & $13(5.8)$ & $18(15.4)$ & $0(0)$ & $0(0)$ & $0(0)$ & $0(0)$ & $0(0)$ \\
\hline
\end{tabular}

a Including serotypes 7A ( $n=1), 7 \mathrm{~B}(n=2), 7 \mathrm{C}(n=1), 12 \mathrm{~F}(n=3), 15 \mathrm{~B}(n=3), 15 \mathrm{~F}(n=2), 17 \mathrm{~F}(n=4), 18 \mathrm{~B}(n=3), 20(n=4), 21(n=2), 22 \mathrm{~F}(n=1), 23 \mathrm{~A}(n=1), 28 \mathrm{~A}(n=1), 29$ $(n=1), 34(n=4), 35 \mathrm{~B}(n=3), 35 \mathrm{~F}(n=1), 37(n=1), 39(n=1)$. 


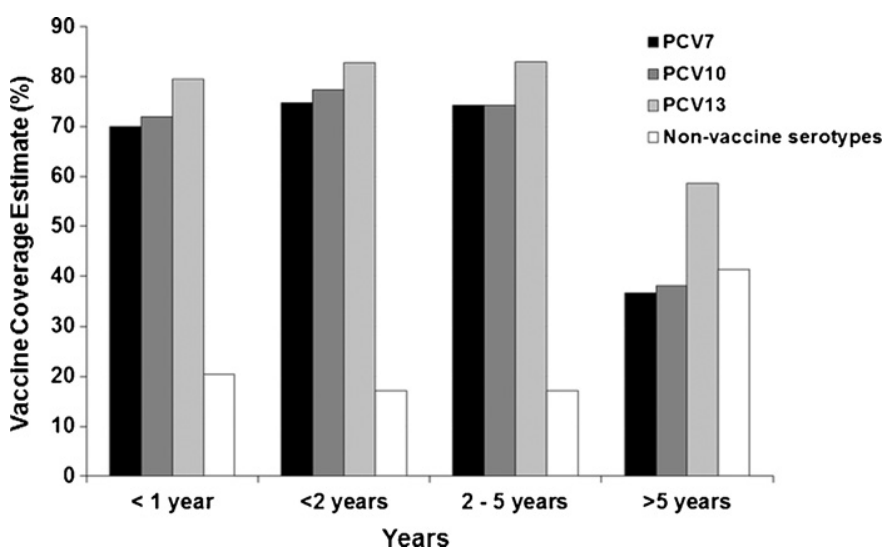

Fig. 1. Estimated projected coverage of the 7-, 10- and 13-valent pneumococcal conjugative vaccines (PCVs) for pneumococcal meningitis cases in Salvador, Brazil, during 2000-2007, according to different age groups.

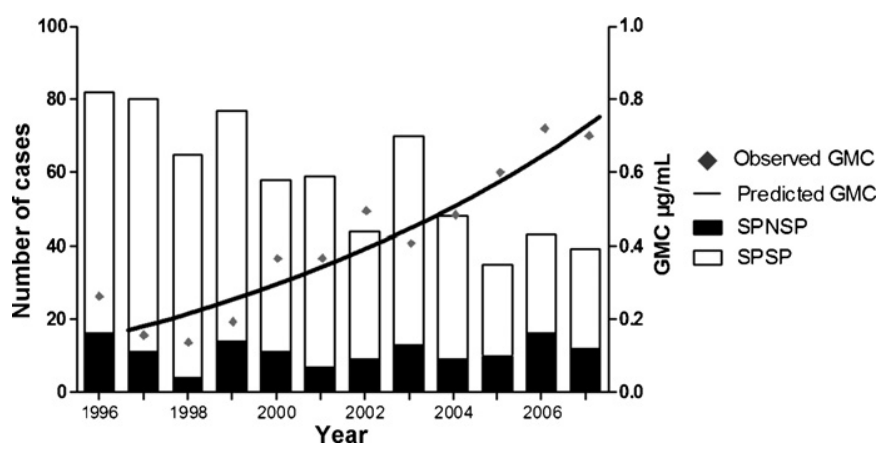

Fig. 2. Trends of geometric mean of penicillin MICs $(\mu \mathrm{g} / \mathrm{mL})$ over time and number of meningitis cases due to penicillin susceptible (SPSP) and non-susceptible (SPNSP) S. pneumoniae isolates, in Salvador, Brazil, 1996-2007.

found among PCV7 isolates. The most common serotypes showing nonsusceptibility to trimethoprim-sulphamethoxazole were serotypes $14(n=47,21.1 \%), 6 \mathrm{~B}(n=26,11.7 \%)$ and $19 \mathrm{~F}(n=24$, $10.8 \%$ ), whereas $15.4 \%$ of serotype $23 \mathrm{~F}(n=18)$ isolates and $11.1 \%$ of serotype $14(n=13)$ isolates were non-susceptible to tetracycline. Four (1\%) serotype 14 isolates were cefotaxime non-susceptible $(\mathrm{MIC}=1 \mu \mathrm{g} / \mathrm{mL})$. This phenotype was associated with resistance to penicillin ( $\mathrm{MIC}=2 \mu \mathrm{g} / \mathrm{mL} ; 2$ isolates and $\mathrm{MIC}=4 \mu \mathrm{g} / \mathrm{mL} ; 2$ isolates $)$ (data not shown).

Overall, 295 (74.3\%) of 397 S. pneumoniae isolates were nonsusceptible to $\geq 1$ drug. Resistance to $\geq 3$ drugs (Sxt-Clo-Tet) was found in one serotype $6 \mathrm{~B}$ isolate. All isolates of serotypes $23 \mathrm{~F}, 5$, $35 \mathrm{~B}, 7 \mathrm{~B}, 21,7 \mathrm{~A}, 7 \mathrm{C}, 22 \mathrm{~F}, 23 \mathrm{~A}, 29,35 \mathrm{~F}$ and 39 , and $\geq 90 \%$ of isolates of the serotypes $6 \mathrm{~A}(n=18), 6 \mathrm{~B}(n=28), 9 \mathrm{~N}(n=10), 10 \mathrm{~A}(n=12), 19 \mathrm{~A}$ $(n=9)$, and $14(n=51)$ were resistant to $\geq 1$ drug (data not shown).

The trend of the geometric mean (GMC) of penicillin MICs during 1996-2007 is displayed in Fig. 2. Despite the decrease in meningitis cases due to penicillin-susceptible $S$. pneumoniae isolates (PSP), a significant increasing trend of GMC was found from 1996 to 2007 $(P=0.0001)$. After 2003, GMCs significantly increased to values as high as $0.25 \mu \mathrm{g} / \mathrm{mL}$ in 2006. Higher penicillin MICs were observed after 2001 (MIC $=2 \mu \mathrm{g} / \mathrm{mL})$ and after $2004(\mathrm{MIC}=4 \mu \mathrm{g} / \mathrm{mL})$ among serotype 14 isolates (data not shown). The penicillin MIC $_{50}$ and $\mathrm{MIC}_{90}$ found during the period of $2000-2007$ were $0.031 \mu \mathrm{g} / \mathrm{mL}$ and $0.25 \mu \mathrm{g} / \mathrm{mL}$, respectively.

\section{Discussion}

Our study describes the serotype distribution and antimicrobial susceptibilities of pneumococcal meningitis isolates collected in Salvador, Brazil during 2000-2007. These results represent an important contribution to our current understanding of the epidemiology and burden of pneumococcal meningitis prior to the introduction of PCV-10. The incidence of pneumococcal infection varies widely in different global regions and is influenced by several key factors such as age and immunization status [25]. Although we have detected a decline in the annual incidence of meningitis cases, in Metropolitan Salvador, from 2000 to 2007, the case-fatality ratio maintained high, particularly among children aged $<5$ years (41.9\%). This finding may be related to the improvement of primary health care of patients and availability of 7-valent pneumococcal vaccine in private clinics since 2002 . Otherwise, the maintenance of high rates of lethality may be related to intrinsic virulence properties of the pathogen and/or immunological conditions of the patients. It is important to point out that the active surveillance was maintained as the same during the period of study, without reduction in the number of sample-taking procedures. These evidences emphasize the need for continual IPD surveillance in our region

Fluctuations in the prevalence of individual serotypes may occur naturally in pneumococcal population in the absence of conjugate vaccine pressure [26]. However, we have observed through comparisons with our earlier surveillance data (29) that the distribution of the major serotypes among S. pneumoniae meningitis isolates has been stable over time in Salvador, with serotype 14 being the predominant serotype. The prevalence of the serotypes $14,6 \mathrm{~B}, 18 \mathrm{C}$, $19 \mathrm{~F}$, and 23F causing meningitis reported by the Regional System of Vaccines (SIREVA), in Latin America, during 2000-2005 [27] also corroborates our surveillance results.

The predicted coverage of PCV7 (74.3\%) and PCV10 (75.7\%) within our study set of cases from children $<5$ years of age varied little, due to the low frequency of serotypes 1,5 , and $7 \mathrm{~F}$ found among these meningitis isolates. However, in respect to PCV13, we observed an expanded covered of $83.1 \%$ in the same group of cases (children $<5$ years), related to the isolation of the serotypes 3 ( $n=1$; $0.7 \%) ; 6 \mathrm{~A}(n=6 ; 4.3 \%)$, and $19 \mathrm{~A}(n=3 ; 2.2 \%)$. The coverage percentages obtained here are in line with those expected for Latin America [27], and higher than those reported from other studies in Brazil, Bangladesh, and United States [28-30].

Since 1996 there has been an increase in penicillin nonsusceptibility of IPD isolates recovered in Salvador, Brazil. Between December 1995 and November 1999 15\% of the S. pneumoniae meningitis isolates were penicillin non-susceptible (MICs, $0.125-1.0 \mu \mathrm{g} / \mathrm{mL}$ ) [17]. The present study shows that the proportion of PNSP increased to 22.2\% during 2000-2007. GMCs show an increasing trend over time, with values as high as $0.25 \mu \mathrm{g} / \mathrm{mL}$ in 2006, and penicillin-resistance was detected. In comparison with our previous study [17], penicillin $\mathrm{MIC}_{50}$ values increased from $0.016 \mu \mathrm{g} / \mathrm{mL}(1996-1999)$ to $0.031 \mu \mathrm{g} / \mathrm{mL}(2000-2007)$, while $\mathrm{MIC}_{90}$ maintained as the same value $(0.25 \mu \mathrm{g} / \mathrm{mL})$.

Most clinically significant antimicrobial resistance is confined to a small number of dominant, so-called "paediatric serotypes": $6 \mathrm{~A}, 6 \mathrm{~B}, 9 \mathrm{~V}, 14,19 \mathrm{~A}, 19 \mathrm{~F}$ and $23 \mathrm{~F}$ [31]. Five of these serotypes are included in PCV10 (6B, 9V, 14, 19F and 23F) [32]. In the present study, $86.4 \%$ of PCV10 isolates were non-susceptible to penicillin, primarily including serotypes $14(n=37,42 \%), 23 \mathrm{~F}(n=19,21.6 \%)$, 6B $(n=10,11.4 \%)$, and $19 \mathrm{~F}(n=9,10.2 \%)$.

The high resistance rates found for trimethoprim/sulphamethoxazole (56\%) and tetracycline (29.6\%) are in agreement with the rates reported in other Brazilian studies $[33,34]$. This observation may be in part associated with antibiotic consumption [35], since these antimicrobial agents are among the most popular drugs, which are sold over the country without medical prescription and are usually administered improperly.

The identification of penicillin-resistant, cefotaxime-nonsusceptible isolates, although few $(n=4 ; 1 \%)$ are of concern. 
Cefotaxime has been the main antibiotic used for treatment of pneumococcal meningitis; however, in areas where beta lactam antibiotic-resistant pneumococcal strains are prevalent cefotaxime associated with vancomycin has been recommended as empirical treatment until susceptibility test results become available [36].

The findings in this report are subject to at least one limitation. The prevalence of serotypes detected during the period of study (2000-2007) and the estimated coverage of the conjugate vaccines are related only for $S$. pneumoniae isolated from meningitis cases. Information regarding other IPD manifestations in our region is often not available. In Brazil, meningitis is a compulsory reportable disease and the bacterial isolation from CSF is routinely performed. This is not the case for other IPD clinical specimens (for example blood or pleural fluid) where mandated diagnostic tests depend upon the physician's decision [30]. Although all of the pneumococcal isolates included in this study originated from only one hospital, this was not a limitation, since Hospital Couto Maia is the state reference hospital in the city of Salvador, with about $95 \%$ of the meningitis reports from the region originating from that site [15]

Serotype replacement with non-vaccine types after widespread use of PCVs remains a potential concern, requiring continual IPD surveillance to identify the emergence of new clinically important strains that can potentially acquire antibiotic resistance [37]. In the present pre-vaccine era we have observed that non-vaccine serotypes were most commonly recovered from patients 5-49 (42.4\%) years and $\geq 50$ (38.2\%) years of age. In the coming years, results of continuous IPD surveillance will monitor the impact of the PCV10 implementation in Brazil. This information will be of great importance for healthcare decisions and further public health interventions that will potentially become necessary for further reducing the IPD burden in Brazil.

\section{Acknowledgements}

Above all, we thank the study patients and their families; and the clinical, laboratory and administrative staff of Hospital Couto Maia. We are especially grateful to Neide O. Silva. Edilane L. Gouveia and Tainara Q. Oliveira for their participation in data collection, processing and analysis. This study was supported by grants from the Brazilian National Research Council (CNPq - 491345/2005-4 and 478685/2007-6), Research Support Foundation for the State of Bahia (FAPESB - 1431040054051 and PP-SUS0001/2009), CAPESPNPD (1472/2008) and Fogarty International Center of the National Institute of Health, USA (R01 TW007303 and D43TW00919).

\section{References}

[1] Lynch 3rd JP, Zhanel GG. Streptococcus pneumoniae: epidemiology, risk factors, and strategies for prevention. Semin Respir Crit Care Med 2009;30(April (2)):189-209.

[2] World Health Organization. Pneumococcal conjugate vaccine for childhood immunization-WHO position paper. Wkly Epidemiol Rec 2007;82(March (12)):93-104.

[3] Levine OS, O'Brien KL, Knoll M, Adegbola RA, Black S, Cherian T, et al. Pneumococcal vaccination in developing countries. Lancet 2006;367(June (9526)):1880-2.

[4] Scott JA. The preventable burden of pneumococcal disease in the developing world. Vaccine 2007;25(March (13)):2398-405.

[5] O’Brien KL, Wolfson LJ, Watt JP, Henkle E, Deloria-Knoll M, McCall N, et al. Burden of disease caused by Streptococcus pneumoniae in children younger than 5 years: global estimates. Lancet 2009;374(September (9693)):893-902.

[6] Hicks LA, Harrison LH, Flannery B, Hadler JL, Schaffner W, Craig AS, et al. Incidence of pneumococcal disease due to non-pneumococcal conjugate vaccine (PCV7) serotypes in the United States during the era of widespread PCV7 vaccination, 1998-2004. J Infect Dis 2007;196(9):1346-54. Nov 1.

[7] Whitney CG, Farley MM, Hadler J, Harrison LH, Bennett NM, Lynfield R, et al. Decline in invasive pneumococcal disease after the introduction of proteinpolysaccharide conjugate vaccine. N Engl J Med 2003;348(May (18)):1737-46.

[8] Munoz-Almagro C, Jordan I, Gene A, Latorre C, Garcia-Garcia JJ, Pallares R. Emergence of invasive pneumococcal disease caused by nonvaccine serotypes in the era of 7-valent conjugate vaccine. Clin Infect Dis 2008;46(2):174-82. Jan 15.

[9] Pilishvili T, Lexau C, Farley MM, Hadler J, Harrison LH, Bennett NM, et al. Sustained reductions in invasive pneumococcal disease in the era of conjugate vaccine. J Infect Dis 2010;201(January (1)):32-41.

[10] Pelton SI, Huot H, Finkelstein JA, Bishop CJ, Hsu KK, Kellenberg J, et al. Emergence of $19 \mathrm{~A}$ as virulent and multidrug resistant Pneumococcus in Massachusetts following universal immunization of infants with pneumococcal conjugate vaccine. Pediatr Infect Dis J 2007;26(June (6)):468-72.

[11] Moore MR, Gertz Jr RE, Woodbury RL, Barkocy-Gallagher GA, Schaffner W, Lexau C, et al. Population snapshot of emergent Streptococcus pneumoniae serotype 19A in the United States, 2005. J Infect Dis 2008;197(April (7)):1016-27.

[12] Prymula R, Schuerman L. 10-Valent pneumococcal nontypeable Haemophilus influenzae PD conjugate vaccine: Synflorix. Expert Rev Vaccines 2009;8(November (11)):1479-500.

[13] Hausdorff WP, Bryant J, Paradiso PR, Siber GR. Which pneumococcal serogroups cause the most invasive disease: implications for conjugate vaccine formulation and use, part I. Clin Infect Dis 2000;30(January (1)):100-21.

[14] PneumoADIP., http://www.vaccineamc.org/files/TPP_Codebook.pdf, 2008.

[15] Secretaria de Saúde do Estado da Bahia. Casos Notificados de Meningite-Bahia; 2008 [cited: Available from: http://www.saude.ba.gov.br/cgi/deftohtm. exe?tabnet/sinan/menin.def].

[16] Ko AI, Reis JN, Coppola SJ, Gouveia EL, Cordeiro SM, Lobo TS, et al. Clonally related penicillin-nonsusceptible Streptococcus pneumoniae serotype 14 from cases of meningitis in Salvador, Brazil. Clin Infect Dis 2000;30(January (1)):78-86.

[17] Reis JN, Cordeiro SM, Coppola SJ, Salgado K, Carvalho MG, Teixeira LM, et al. Population-based survey of antimicrobial susceptibility and serotype distribution of Streptococcus pneumoniae from meningitis patients in Salvador, Brazil. J Clin Microbiol 2002;40(January (1)):275-7.

[18] Arbique JC, Poyart C, Trieu-Cuot P, Quesne G, Carvalho Mda G, Steigerwalt AG, et al. Accuracy of phenotypic and genotypic testing for identification of Streptococcus pneumoniae and description of Streptococcus pseudopneumoniae sp. nov. J Clin Microbiol 2004;42(October (10)):4686-96.

[19] Pai R, Gertz RE, Beall B. Sequential multiplex PCR approach for determining capsular serotypes of Streptococcus pneumoniae isolates. J Clin Microbiol 2006;44(January (1)):124-31.

[20] Dias CA, Teixeira LM, Carvalho Mda G, Beall B. Sequential multiplex PCR for determining capsular serotypes of pneumococci recovered from Brazilian children. J Med Microbiol 2007;56(September (Pt. 9)):1185-8.

[21] Centers for Disease Control Prevention. PCR deduction of pneumococcal serotypes; March 2010 [cited: Available from: http://www.cdc.gov/ncidod/ biotech/strep/pcr.htm]

[22] Campos LC, Carvalho Mda G, Beall BW, Cordeiro SM, Takahashi D, Reis MG, et al. Prevalence of Streptococcus pneumoniae serotype 6C among invasive and carriage isolates in metropolitan Salvador, Brazil, from 1996 to 2007. Diagn Microbiol Infect Dis 2009;65(October (2)):112-5.

[23] Clinical and Laboratory Standards Institute. Performance Standards for Antimicrobial Susceptibility Testing. Seventeenth Informational Supplement. Wayne, PA: Approved Standard M100-S17; 2007.

[24] Instituto Brasileiro de Geografia e Estatística. Censo Demografico 2000-Resultados do universo. Rio de Janeiro: Instituto Brasileiro de Geografia e Estatística; 2002.

[25] Hausdorff WP, Feikin DR, Klugman KP. Epidemiological differences among pneumococcal serotypes. Lancet Infect Dis 2005;5(February (2)):83-93.

[26] Ruckinger S, von Kries R, Reinert RR, van der Linden M, Siedler A. Childhood invasive pneumococcal disease in Germany between 1997 and 2003: variability in incidence and serotype distribution in absence of general pneumococcal conjugate vaccination. Vaccine 2008;26(July (32)):3984-6.

[27] Castaneda E, Agudelo CI, Regueira M, Corso A, Brandileone MC, Brandao AP, et al. Laboratory-based surveillance of Streptococcus pneumoniae invasive disease in children in 10 Latin American countries: a SIREVA II project, 2000-2005. Pediatr Infect Dis J 2009;28(September (9)):e265-70.

[28] Hsu KK, Shea KM, Stevenson AE, Pelton SI. Changing serotypes causing childhood invasive pneumococcal disease: Massachusetts, 2001-2007. Pediatr Infect Dis J 2010;29(April (4)):289-93.

[29] Saha SK, Naheed A, El Arifeen S, Islam M, Al-Emran H, Amin R, et al. Surveillance for invasive Streptococcus pneumoniae disease among hospitalized children in Bangladesh: antimicrobial susceptibility and serotype distribution. Clin Infect Dis 2009;48(March (2)):S75-81.

[30] Brandileone MC, de Andrade AL, Di Fabio JL, Guerra ML, Austrian R. Appropriateness of a pneumococcal conjugate vaccine in Brazil: potential impact of age and clinical diagnosis, with emphasis on meningitis. J Infect Dis 2003;187(April 8):1206-12.

[31] Klugman KP. The successful clone: the vector of dissemination of resistance in Streptococcus pneumoniae. J Antimicrob Chemother 2002;50(December $(\mathrm{S} 2)): 1-5$.

[32] Dagan R. Impact of pneumococcal conjugate vaccine on infections caused by antibiotic-resistant Streptococcus pneumoniae. Clin Microbiol Infect 2009;15(April (3)):16-20.

[33] Koeth LM, Felmingham D, Jacobs MR, Rossi F. Antimicrobial resistance of Streptococcus pneumoniae and Haemophilus influenzae in Sao Paulo, Brazil from 1996 to 2000. Int J Antimicrob Agents 2004;23(April (4)):356-61.

[34] Brandileone MC, Casagrande ST, Guerra ML, Zanella RC, Andrade AL, Di Fabio JL. Increase in numbers of beta-lactam-resistant invasive Streptococcus pneumo- 
niae in Brazil and the impact of conjugate vaccine coverage. J Med Microbiol 2006;55(May (Pt. 5)):567-74.

[35] Goossens H. Antibiotic consumption and link to resistance. Clin Microbiol Infect 2009;15(April (Suppl. 3)):12-5.
[36] Kim KS. Acute bacterial meningitis in infants and children. Lancet Infect Dis 2010;10(January (1)):32-42.

[37] Klugman KP, Garau J. A preventable killer: pneumonia. Clin Microbiol Infect 2009;15(November (11)):989-90. 